\title{
Polynomial algebras and exact solutions of general quantum non-linear optical models I: Two-mode boson systems
}

\author{
Yuan-Harng Lee ${ }^{a}$, Wen-Li Yang ${ }^{b}$ and Yao-Zhong Zhang ${ }^{a}$ \\ a. School of Mathematics and Physics, The University of Queensland, Brisbane, Qld \\ 4072, Australia \\ b. Institute of Modern Physics, Northwest University, Xi'an 710069, China
}

\begin{abstract}
We introduce higher order polynomial deformations of $A_{1}$ Lie algebra. We construct their unitary representations and the corresponding single-variable differential operator realizations. We then use the results to obtain exact (Bethe ansatz) solutions to a class of 2-mode boson systems, including the Boson-Einstein Condensate models as special cases. Up to an overall factor, the eigenfunctions of the 2-mode boson systems are given by polynomials whose roots are solutions of the associated Bethe ansatz equations. The corresponding eigenvalues are expressed in terms of these roots. We also establish the spectral equivalence between the BEC models and certain quasi-exactly solvable Schördinger potentials.
\end{abstract}

PACS numbers: 02.20.-a; 02.20.Sv; 03.65.Fd; 42.65.Ky.

Keywords: Polynomial algebras, quasi-exactly solvable models, Bethe ansatz.

\section{Introduction}

Polynomial algebras are non-linear deformations of Lie algebras and have recently found widespread applications in theoretical physics whereby they appear in diverse topics such as quantum mechanics, Yang-Mills type gauge theories, quantum non-linear optics, integrable systems and (quasi-)exactly solvable models, to name a few (see e.g. [1]-[10]).

One of the reasons for their increasing prevalence stems from the realization that traditional linear Lie algebras describe only a very restrictive subset of linear symmetries and that many physical systems do in fact possess non-linear symmetries such as those in which the commutations of the symmetry algebra generators return polynomial terms.

Due to their importance, a number of studies have been undertaken to investigate the mathematical properties of these algebras [11]. In particular, differential realizations of 
certain quadratic and cubic algebras have been explored in $[12,13]$ and also in $[14,15]$ in connection with the theory of quasi-exact integrability [16, 17, 18].

In this article, we introduce a novel class of higher order polynomial deformations of the classical $A_{1}$ Lie algebra and construct their unitary representations in terms of boson operators and single-variable differential operators. We will then use the differential realizations of these algebras and the Functional Bethe Ansatz method (see e.g. [19, 20]) to obtain one of the main results of this paper, that is the exact eigenfunctions and energy eigenvalues of the following class of Hamiltonians

$$
H=\sum_{i}^{2} w_{i} N_{i}+\sum_{i, j}^{2} w_{i j} N_{i} N_{j}+g\left(a_{1}^{\dagger s} a_{2}^{r}+a_{1}^{s} a_{2}^{\dagger r}\right), \quad r, s \in \mathbf{N},
$$

where and throughout $a_{i}\left(a_{i}^{\dagger}\right)$ are boson or photon annihilation (creation) operators with frequencies $w_{i}, N_{i}=a_{i}^{\dagger} a_{i}$ are number operators, $w_{i j}$ and $g$ are real coupling constants. Without loss of generality, in the following we will identify $w_{12}$ with $w_{21}$. Hamiltonians (1.1) appear in the description of various physical systems of interest such as non-linear optics [8, 9] and Bose-Einstein Condensates (BECs) [21]-[24]. For instance, the nondiagonal terms in (1.1) describe processes of multi-photon scattering and higher-order harmonic generation in quantum nonlinear optics. Let us point out that (1.1) is a twomode version of the more general multi-mode Hamiltonian considered in [25] in which the quasi-exact solvability of the multi-mode system was established by a different procedure and without giving exact solutions (see also [26] for the case of third-order harmonic generation)* Hamiltonians for the special cases of $s=r=1$ and $s=2, r=1$ have also been studied using Algebraic Bethe Ansatz (ABA) method [27].

This paper is organized as follows, in section 2 we propose a class of generalized polynomial $s u(1,1)$ algebras and derive their boson realizations. In section 3 , we use these deformed $s u(1,1)$ algebras as base elements to generate higher order polynomial algebras via a Jordan-Schwinger like construction method. We then identify these algebras as the dynamical algebra of the Hamiltonian (1.1) in section 4 and solve for the eigenvalue problem in general via the Functional Bethe Ansatz method. In section 5, we present explicit results for the Hamiltonian (1.1) when $r, s \leq 2$ and $r=s=3$. In section 6 , we establish the spectral correspondence of these specific models with quasi-exactly solvable (QES) Schrödinger potentials. Finally we summarize our results in section 7 and discuss further avenues of investigation.

${ }^{*}$ We became aware of these three references after submitting our work. We thank one referee for pointing them out. 


\section{Polynomial deformations of $\operatorname{su}(1,1)$ algebra}

Let $k$ be a positive integer, $k=1,2, \cdots$. We start off by proposing a class of polynomial algebras of degree $k-1$ defined by the commutation relations

$$
\begin{aligned}
{\left[Q_{0}, Q_{ \pm}\right] } & = \pm Q_{ \pm} \\
{\left[Q_{+}, Q_{-}\right] } & =\phi^{(k)}\left(Q_{0}\right)-\phi^{(k)}\left(Q_{0}-1\right)
\end{aligned}
$$

where

$$
\phi^{(k)}\left(Q_{0}\right)=-\prod_{i=1}^{k}\left(Q_{0}+\frac{i}{k}-\frac{1}{k^{2}}\right)+\prod_{i=1}^{k}\left(\frac{i-k}{k}-\frac{1}{k^{2}}\right)
$$

is a $k^{t h}$-order polynomial in $Q_{0}$. The algebra admits Casimir operator of the following form

$$
C=Q_{-} Q_{+}+\phi^{(k)}\left(Q_{0}\right)=Q_{+} Q_{-}+\phi^{(k)}\left(Q_{0}-1\right) .
$$

For $k=1$ and $k=2,(2.1)$ reduces to the oscillator and $s u(1,1)$ algebras, respectively. Thus, the algebra (2.1) can be viewed as polynomial extensions of the linear $s u(1,1)$ and oscillator algebras.

Similar to the $s u(1,1)$ algebra case, unitary representations of $(2.1)$ are infinite dimensional. In this section, we shall concentrate on the following one-mode boson realization of the algebra,

$$
Q_{+}=\frac{1}{(\sqrt{k})^{k}}\left(a^{\dagger}\right)^{k}, \quad Q_{-}=\frac{1}{(\sqrt{k})^{k}}(a)^{k}, \quad Q_{0}=\frac{1}{k}\left(a^{\dagger} a+\frac{1}{k}\right) .
$$

In this realization, the Casimir (2.3) takes the particular value,

$$
C=\prod_{i=1}^{k}\left(\frac{i-k}{k}-\frac{1}{k^{2}}\right) .
$$

We now construct the unitary representations corresponding to the realization $(2.4)$ in the Fock space $\mathcal{H}_{b}$. There are $k$ lowest weight states,

$$
|0\rangle, \quad\left(a^{\dagger}\right)|0\rangle, \ldots, \quad\left(a^{\dagger}\right)^{k-1}|0\rangle .
$$

Writing these lowest weight states as $|q, 0\rangle$ using the Bargmann index $q$, we have

$$
Q_{0}|q, 0\rangle=q|q, 0\rangle, \quad Q_{-}|q, 0\rangle=0
$$

It follows from (2.3) and (2.5) that $\prod_{i=1}^{k}\left(q+\frac{i-k}{k}-\frac{1}{k^{2}}\right)=0$, from which we get

$$
q=\frac{1}{k^{2}}, \frac{k+1}{k^{2}}, \frac{2 k+1}{k^{2}}, \cdots, \frac{(k-1) k+1}{k^{2}} .
$$

This means that the boson realization (2.4) corresponds to the infinite dimensional unitary representation with particular $q$ values (2.8). In other words, the $\mathcal{H}_{b}$ decomposes into the direct $\operatorname{sum} \mathcal{H}_{b}=\mathcal{H}_{b}^{\frac{1}{k^{2}}} \oplus \cdots \oplus \mathcal{H}_{b}^{\frac{(k-1) k+1}{k^{2}}}$ of $k$ irreducible components $\mathcal{H}_{b}^{\frac{1}{k^{2}}}, \ldots, \mathcal{H}_{b}^{\frac{(k-1) k+1}{k^{2}}}$. 
Noting $k q-\frac{1}{k}=0,1, . ., k-1$ for all the allowed $q$ values given in (2.8), we can write the lowest weight states $(2.6)$ as $|q, 0\rangle=\left(a^{\dagger}\right)^{k q-\frac{1}{k}}|0\rangle$. The general Fock states $|q, n\rangle \sim Q_{+}^{n}|q, 0\rangle$ in the irreducible representation space $\mathcal{H}_{b}^{q}$ are then given by

$$
|q, n\rangle=\frac{a^{\dagger k\left(n+q-\frac{1}{k^{2}}\right)}}{\sqrt{\left[k\left(n+q-\frac{1}{k^{2}}\right)\right] !}}|0\rangle .
$$

It is easy to show that $Q_{0}, Q_{ \pm}$and $C$ act on these states as follows

$$
\begin{aligned}
Q_{0}|q, n\rangle & =(q+n)|q, n\rangle, \\
Q_{+}|q, n\rangle & =\prod_{i=1}^{k}\left(n+q+\frac{i k-1}{k^{2}}\right)^{\frac{1}{2}}|q, n+1\rangle, \\
Q_{-}|q, n\rangle & =\prod_{i=1}^{k}\left(n+q-\frac{(i-1) k+1}{k^{2}}\right)^{\frac{1}{2}}|q, n-1\rangle, \\
C|q, n\rangle & =\prod_{i=1}^{k}\left(\frac{i-k}{k}-\frac{1}{k^{2}}\right)|q, n\rangle, \\
n & =0,1, \cdots, \quad q=\frac{1}{k^{2}}, \frac{k+1}{k^{2}}, \cdots, \frac{(k-1) k+1}{k^{2}} .
\end{aligned}
$$

\section{Polynomial algebras via Jordan-Schwinger type con- struction}

The unitary representations of the polynomial algebras discussed in the preceding section are all infinite dimensional. In this section, we shall employ a Jordan-Schwinger like construction $[12,13]$, to derive polynomial algebras that have finite dimensional unitary representations. Towards this end, we consider two mutually commuting polynomial algebras introduced in the preceding section, $\left\{Q_{+}^{(1)}, Q_{-}^{(1)}, Q_{0}^{(1)}\right\}$ of degree $\left(k_{1}-1\right)$ and $\left\{Q_{+}^{(2)}, Q_{-}^{(2)}, Q_{0}^{(2)}\right\}$ of degree $\left(k_{2}-1\right)$, where $k_{1}, k_{2}=1,2, \cdots$.

Introduce new generators,

$$
\mathcal{Q}_{+}=Q_{+}^{(1)} Q_{-}^{(2)}, \quad \mathcal{Q}_{-}=Q_{+}^{(2)} Q_{-}^{(1)}, \quad \mathcal{Q}_{0}=\frac{1}{2}\left(Q_{0}^{(1)}-Q_{0}^{(2)}\right)
$$

We can easily show that $\mathcal{Q}_{0, \pm}$ form a polynomial algebra of degree $\left(k_{1}+k_{2}-1\right)$ which close under the following commutation relations:

$$
\begin{aligned}
{\left[\mathcal{Q}_{0}, \mathcal{Q}_{ \pm}\right] } & = \pm \mathcal{Q}_{ \pm} \\
{\left[\mathcal{Q}_{+}, \mathcal{Q}_{-}\right] } & =\varphi^{\left(k_{1}+k_{2}\right)}\left(\mathcal{Q}_{0}, \mathcal{L}\right)-\varphi^{\left(k_{1}+k_{2}\right)}\left(\mathcal{Q}_{0}-1, \mathcal{L}\right)
\end{aligned}
$$

where

$$
\mathcal{L}=\frac{1}{2}\left(Q_{0}^{(1)}+Q_{0}^{(2)}\right)
$$


is the central element of the algebra,

$$
\left[\mathcal{L}, \mathcal{Q}_{ \pm, 0}\right]=0
$$

and

$$
\varphi^{\left(k_{1}+k_{2}\right)}\left(\mathcal{Q}_{0}, \mathcal{L}\right)=-\prod_{i=1}^{k_{1}}\left(\mathcal{L}+\mathcal{Q}_{0}+\frac{i}{k_{1}}-\frac{1}{k_{1}^{2}}\right) \prod_{j=1}^{k_{2}}\left(\mathcal{L}-\left(\mathcal{Q}_{0}+1\right)+\frac{j}{k_{2}}-\frac{1}{k_{2}^{2}}\right)
$$

is a $\left(k_{1}+k_{2}\right)^{t h}$-order polynomial in $Q_{0}$ and the central elements $\mathcal{L}$. The Casimir operator of (3.2) is given by

$$
\mathcal{C}=\mathcal{Q}_{-} \mathcal{Q}_{+}+\varphi^{\left(k_{1}+k_{2}\right)}\left(\mathcal{Q}_{0}, \mathcal{L}\right)=\mathcal{Q}_{+} \mathcal{Q}_{-}+\varphi^{\left(k_{1}+k_{2}\right)}\left(\mathcal{Q}_{0}-1, \mathcal{L}\right)
$$

For $k_{1}+k_{2}=2$, i.e. $k_{1}=k_{2}=1$, the polynomial algebra (3.2) reduces to the linear $s u(2)$ algebra. So the algebras defined by (3.2) are polynomial deformations of $s u(2)$.

In terms of two sets of mutually commuting boson operators acting on the tensor product of the Fock spaces, we have the realization $(i=1,2$ below)

$$
Q_{+}^{(i)}=\frac{1}{\left(\sqrt{k_{i}}\right)^{k_{i}}}\left(a_{2}^{\dagger}\right)^{k_{i}}, \quad Q_{-}^{(i)}=\frac{1}{\left(\sqrt{k_{i}}\right)^{k_{i}}}\left(a_{2}\right)^{k_{i}}, \quad Q_{0}^{(i)}=\frac{1}{k_{i}}\left(N_{i}+\frac{1}{k_{i}}\right) .
$$

This realization gives rise to finite dimensional representations of the polynomial algebra (3.2). To show this, let $\left|q_{1}, n_{1}\right\rangle,\left|q_{2}, n_{2}\right\rangle$ be the one-mode Fock states of the algebras $\left\{Q_{0, \pm}^{(1)}\right\},\left\{Q_{0, \pm}^{(2)}\right\}$ respectively, where $n_{1}, n_{2}=0,1, \cdots$, and $q_{1}=\frac{1}{k_{1}^{2}}, \frac{k_{1}+1}{k_{1}^{2}}, \cdots, \frac{\left(k_{1}-1\right) k_{1}+1}{k_{1}^{2}}$ and $q_{2}=\frac{1}{k_{2}^{2}}, \frac{k_{2}+1}{k_{2}^{2}}, \cdots, \frac{\left(k_{2}-1\right) k_{2}+1}{k_{2}^{2}}$. The representations of $\left\{\mathcal{Q}_{0, \pm}\right\}$ corresponding to the realization (3.7) are then given by the two-mode Fock states $\left|q_{1}, n_{1}\right\rangle\left|q_{2}, n_{2}\right\rangle$. Since $\mathcal{L}$ is a central element of the algebra, it must be a constant, denoted as $l$ below, on any irreducible representations. This imposes a constraint on the values of $n_{1}$ and $n_{2}$,

$$
\begin{aligned}
\mathcal{L}\left|q_{1}, n_{1}\right\rangle\left|q_{2}, n_{2}\right\rangle & =\frac{1}{2}\left(q_{1}+n_{1}+q_{2}+n_{2}\right)\left|q_{1}, n_{1}\right\rangle\left|q_{2}, n_{2}\right\rangle \\
& =l\left|q_{1}, n_{1}\right\rangle\left|q_{2}, n_{2}\right\rangle .
\end{aligned}
$$

That is $n_{1}+n_{2}=2 l-\left(q_{1}+q_{2}\right)$. Thus obviously $2 l-q_{1}-q_{2}$ take only positive integer values, i.e.

$$
2 l-q_{1}-q_{2}=0,1, \cdots .
$$

It follows that the Fock states corresponding to the realization (3.7) are

$$
\begin{aligned}
\left|q_{1}, q_{2}, n, l\right\rangle & =\left|q_{1}, n\right\rangle\left|q_{2}, 2 l-q_{1}-q_{2}-n\right\rangle \\
& =\frac{\left(a_{1}^{\dagger}\right)^{k_{1}\left(n+q_{1}-\frac{1}{k_{1}^{2}}\right)}\left(a_{2}^{\dagger}\right)^{k_{2}\left(2 l-q_{1}-n-\frac{1}{k_{2}^{2}}\right)}}{\sqrt{\left(k_{1}\left(n+q_{1}-\frac{1}{k_{1}^{2}}\right)\right) !} \sqrt{\left(k_{2}\left(2 l-q_{1}-\frac{1}{k_{2}^{2}}-n\right)\right) !}}|0\rangle \\
n & =0,1, \cdots, 2 l-q_{1}-q_{2},
\end{aligned}
$$


noting that $2 l-q_{1}-q_{2}$ is always less than or equal to $2 l-q_{1}-\frac{1}{k_{2}^{2}}$. This gives us the $2 l-q_{1}-q_{2}+1$ dimensional irreducible representation of (3.2),

$$
\begin{aligned}
\mathcal{Q}_{0}\left|q_{1}, q_{2}, n, l\right\rangle= & \left(q_{1}-l+n\right)\left|q_{1}, q_{2}, n, l\right\rangle, \\
\mathcal{Q}_{+}\left|q_{1}, q_{2}, n, l\right\rangle= & \prod_{i=1}^{k_{2}}\left(2 l-q_{1}-n-\frac{k_{2}(i-1)+1}{k_{2}^{2}}\right)^{\frac{1}{2}} \\
& \times \prod_{j=1}^{k_{1}}\left(n+q_{1}+\frac{j k_{1}-1}{k_{1}^{2}}\right)^{\frac{1}{2}}\left|q_{1}, q_{2}, n+1, l\right\rangle, \\
\mathcal{Q}_{-}\left|q_{1}, q_{2}, n, l\right\rangle= & \prod_{i=1}^{k_{2}}\left(2 l-q_{1}-n+\frac{i k_{2}-1}{k_{2}^{2}}\right)^{\frac{1}{2}} \\
& \times \prod_{j=1}^{k_{1}}\left(n+q_{1}-\frac{(j-1) k_{1}+1}{k_{1}^{2}}\right)^{\frac{1}{2}}\left|q_{1}, q_{2}, n-1, l\right\rangle .
\end{aligned}
$$

By using the Fock-Bargmann correspondence,

$$
a_{i}^{\dagger} \longrightarrow z_{i}, \quad a_{i} \longrightarrow \frac{d}{d z_{i}}, \quad\left|n_{i}\right\rangle \longrightarrow \frac{z_{i}^{n_{i}}}{\sqrt{n_{i} !}}
$$

we can make the following association

$$
\left|q_{1}, q_{2}, n, l\right\rangle \longrightarrow \frac{z_{1}^{k_{1}\left(n+q_{1}-\frac{1}{k_{1}^{2}}\right)} k_{2} k_{2}\left(2 l-q_{1}-n-\frac{1}{k_{2}^{2}}\right)}{\sqrt{\left(k_{1}\left(n+q_{1}-\frac{1}{k_{1}^{2}}\right)\right) !} \cdot \sqrt{\left(k_{2}\left(2 l-q_{1}-\frac{1}{k_{2}^{2}}-n\right)\right) !}}
$$

Now since $l, q_{1}, q_{2}, k_{1}, k_{2}$ are constants, we can map the states $\left|q_{1}, q_{2}, n, l\right\rangle$ above to the monomials in $z=z_{1}^{k_{1}} / z_{2}^{k_{2}}$,

$$
\begin{aligned}
\Psi_{q_{1}, q_{2}, n, l}(z) & =\frac{z^{n}}{\sqrt{\left(k_{1}\left(n+q_{1}-\frac{1}{k_{1}^{2}}\right)\right) !} \sqrt{\left(k_{2}\left(2 l-q_{1}-\frac{1}{k_{2}^{2}}-n\right)\right) !}}, \\
n & =0,1, \cdots, 2 l-q_{1}-q_{2} .
\end{aligned}
$$

The corresponding single-variable differential operator realization of (3.2) takes the following form

$$
\begin{aligned}
\mathcal{Q}_{0} & =z \frac{d}{d z}+q_{1}-l \\
\mathcal{Q}_{+} & =z \frac{\left(\sqrt{k_{2}}\right)^{k_{2}}}{\left(\sqrt{k_{1}}\right)^{k_{1}}} \prod_{j=1}^{k_{2}}\left(2 l-q_{1}-\frac{(j-1) k_{2}+1}{k_{2}^{2}}-z \frac{d}{d z}\right), \\
\mathcal{Q}_{-} & =z^{-1} \frac{\left(\sqrt{k_{1}}\right)^{k_{1}}}{\left(\sqrt{k_{2}}\right)^{k_{2}}} \prod_{j=1}^{k_{1}}\left(z \frac{d}{d z}+q_{1}-\frac{(j-1) k_{1}+1}{k_{1}^{2}}\right) .
\end{aligned}
$$

These differential operators form the same $2 l-q_{1}-q_{2}+1$ dimensional representations in the space of polynomials as those realized by (3.7) in the corresponding Fock space. We remark that because $\prod_{j=1}^{k_{1}}\left(q_{1}-\frac{(j-1) k_{1}+1}{k_{1}^{2}}\right) \equiv 0$ for all the allowed $q_{1}$ values there is no $z^{-1}$ term in $\mathcal{Q}_{-}$above and thus the differential operator expressions (3.15) are non-singular. 


\section{Exact solution of the 2-mode boson systems}

We now use the differential operator realization (3.15) to exactly solve the 2-mode boson Hamiltonian (1.1).

By means of the Jordan-Schwinger type construction (3.1) and the realization (3.7), identifying $k_{1}$ with $s$ and $k_{2}$ with $r$, we may express the Hamiltonian (1.1) in terms of the generators of the polynomial algebra (3.2),

$$
H=\sum_{i}^{2} w_{i} N_{i}+\sum_{i, j}^{2} w_{i j} N_{i} N_{j}+g \sqrt{s^{s} r^{r}}\left(\mathcal{Q}_{+}+\mathcal{Q}_{-}\right)
$$

with the number operators having the following expressions in $\mathcal{Q}_{0}$ and $\mathcal{L}$

$$
N_{1}=s\left(\mathcal{Q}_{0}+\mathcal{L}\right)-\frac{1}{s}, \quad N_{2}=r\left(\mathcal{L}-\mathcal{Q}_{0}\right)-\frac{1}{r} .
$$

Keep in mind that $\left\{\mathcal{Q}_{\mp, 0}\right\}$ in (4.1) as realized by (3.7) (and (3.1)) form the $\left(2 l-q_{1}-q_{2}\right)+1$ dimensional representation of the polynomial algebra (3.2). This representation is also realized by the differential operators $(3.15)$ acting on the $\left(2 l-q_{1}-q_{2}\right)+1$ dimensional space of polynomials with basis $\left\{1, z, z^{2}, \ldots, z^{2 l-q_{1}-q_{2}}\right\}$. We can thus equivalently represent (4.1) (i.e. (1.1)) as the single-variable differential operator of order $\max \{s, r, 2\}$,

$$
\begin{aligned}
H= & \sum_{i}^{2} w_{i} N_{i}+\sum_{i, j}^{2} w_{i j} N_{i} N_{j} \\
& +g z \prod_{j=1}^{r} r\left(2 l-q_{1}-\frac{(j-1) r+1}{r^{2}}-z \frac{d}{d z}\right) \\
& +g z^{-1} \prod_{j=1}^{s} s\left(z \frac{d}{d z}+q_{1}-\frac{(j-1) s+1}{s^{2}}\right)
\end{aligned}
$$

with

$$
N_{1}=s\left(z \frac{d}{d z}+q_{1}\right)-\frac{1}{s}, \quad N_{2}=r\left(2 l-q_{1}-z \frac{d}{d z}\right)-\frac{1}{r} .
$$

We will now solve for the Hamiltonian equation

$$
H \psi(z)=E \psi(z)
$$

by using the Functional Bethe Ansatz method, where $\psi(z)$ is the eigenfunction and $E$ is the corresponding eigenvalue. It is easy to verify

$$
H z^{m}=z^{m+1} g \prod_{j=1}^{r} r\left(2 l-q_{1}-\frac{(j-1) r+1}{r^{2}}-m\right)+\text { lower order terms, } \quad m \in \mathbf{Z}_{+} .
$$

This means that the differential operator (4.3) is not exactly solvable. However, it is quasi exactly solvable, since it has an invariant polynomial subspace of degree $\left(2 l-q_{1}-q_{2}\right)+1$ :

$$
H \mathcal{V} \subseteq \mathcal{V}, \quad \mathcal{V}=\operatorname{span}\left\{1, z, \ldots, z^{2 l-q_{1}-q_{2}}\right\}, \quad \operatorname{dim} \mathcal{V}=2 l-q_{1}-q_{2}+1
$$


This is easily seen from the fact that when $m=2 l-q_{1}-q_{2}$ the first term on the r.h.s. of (4.6) becomes $z^{2 l-q_{1}-q_{2}+1} g \prod_{j=1}^{r} r\left(q_{2}-\frac{(j-1) r+1}{r^{2}}\right)$ which vanishes identically for all the allowed $q_{2}$ values. We remark that the quasi-exact solvability of the system is connected with its quantum integrability, i.e. with the fact that there exists quantum operator coinciding with a linear combination of the operators $N_{1}$ and $N_{2}$ which commutes with the Hamiltonian (1.1).

As (4.3) is a quasi exactly solvable differential operator preserving $\mathcal{V}$, up to an overall factor, its eigenfunctions have the form,

$$
\psi(z)=\prod_{i=1}^{M}\left(z-\alpha_{i}\right)
$$

where $M \equiv 2 l-q_{1}-q_{2}(=0,1, \cdots)$, and $\left\{\alpha_{i} \mid i=1,2, \cdots, M\right\}$ are roots of the polynomial which will be specified later by the associated Bethe ansatz equations (4.14) below. We can rewrite the Hamiltonian (4.3) as

$$
H=\sum_{i=1}^{\max \{r, s, 2\}} P_{i}(z)\left(\frac{d}{d z}\right)^{i}+P_{0}(z)
$$

where

$$
\begin{aligned}
P_{0}(z)= & z g \prod_{i=1}^{r} r\left(2 l-q_{1}-\frac{(i-1) r+1}{r^{2}}\right) \\
& +w_{11}\left(s q_{1}-\frac{1}{s}\right)^{2}+w_{22}\left(r\left(2 l-q_{1}\right)-\frac{1}{r}\right)^{2} \\
& +2 w_{12}\left(s q_{1}-\frac{1}{s}\right)\left(r\left(2 l-q_{1}\right)-\frac{1}{r}\right) \\
& +w_{1}\left(s q_{1}-\frac{1}{s}\right)+w_{2}\left(r\left(2 l-q_{1}\right)-\frac{1}{r}\right)
\end{aligned}
$$

and $P_{i}(z)$ are the coefficients in front of $d^{i} / d z^{i}$ in the expansion of (4.3) (see the Appendix),

$$
\begin{aligned}
P_{i}(z)= & g s^{s} z^{i-1} \sum_{k=i}^{s}\left(\sum_{l_{1}<\ldots<l_{k}}^{s} \prod_{j \neq l_{1} \neq \cdots \neq l_{k}}^{s} A_{j}\right) L_{k, i} \\
& +g(-r)^{r} z^{i+1} \sum_{k=i}^{r}\left(\sum_{l_{1}<\ldots<l_{k}}^{r} \prod_{j \neq l_{1} \neq \cdots \neq l_{k}}^{r} B_{j}\right) L_{k, i} \\
& +F \delta_{i, 2} z^{2}+D \delta_{i, 1} z .
\end{aligned}
$$

In the above expression,

$$
\begin{aligned}
A_{i} & =q_{1}-\frac{(i-1) s+1}{s^{2}} \\
B_{i} & =-\left(2 l-q_{1}-\frac{(i-1) r+1}{r^{2}}\right), \\
L_{k, k} & =1,
\end{aligned}
$$




$$
\begin{aligned}
L_{k, i}= & \sum_{n_{1}<\ldots<n_{k-i}}^{k-1} n_{1}\left(n_{2}-1\right) \ldots\left(n_{k-i}-(k-i)+1\right), \quad i<k \\
F= & w_{22} r^{2}+w_{11} s^{2}-2 w_{12} s r \\
D= & w_{22} r^{2}\left(1-2\left(2 l-q_{1}-\frac{1}{r^{2}}\right)\right)+w_{11} s^{2}\left(1+2\left(q_{1}-\frac{1}{s^{2}}\right)\right) \\
& +2 w_{12} r s\left(2\left(l-q_{1}\right)+\frac{1}{s^{2}}-\frac{1}{r^{2}}-1\right)+w_{1} s-w_{2} r .
\end{aligned}
$$

Dividing the Hamiltonian equation $H \psi=E \psi$ over by $\psi$ gives us

$$
E=\frac{H \psi}{\psi}=\sum_{i=1}^{\max \{r, s, 2\}} P_{i}(z) i ! \sum_{l_{1}<l_{2}<\ldots<l_{i}}^{M} \frac{1}{\left(z-\alpha_{l_{1}}\right) \ldots\left(z-\alpha_{l_{i}}\right)}+P_{0}(z) .
$$

The l.h.s. of (4.13) is a constant, while the r.h.s is a meromorphic function in $z$ with at most simple poles. For them to be equal, we need to eliminate all singularities on the r.h.s of (4.13). We may achieve this by demanding that the residues of the simple poles, $z=\alpha_{i}, i=1,2, \ldots, M$ should all vanish. This leads to the Bethe ansatz equations for the roots $\left\{\alpha_{i}\right\}$ :

$$
\sum_{i=2}^{\max \{r, s, 2\}} \sum_{l_{1}<l_{2}<\ldots<l_{i-1} \neq p}^{M} \frac{P_{i}\left(\alpha_{p}\right) i !}{\left(\alpha_{p}-\alpha_{l_{1}}\right) \cdots\left(\alpha_{p}-\alpha_{l_{i-1}}\right)}+P_{1}\left(\alpha_{p}\right)=0, \quad p=1,2, \cdots, M .
$$

The wavefunction $\psi(z)$ (4.8) becomes the eigenfunction of $H(4.3)$ in the space $\mathcal{V}$ provided that the roots $\left\{\alpha_{i}\right\}$ of the polynomial $\psi(z)$ (4.8) are the solutions of (4.14).

Some remarks are in order. It is easily seen (from (4.8) and (4.15) below) that $H \psi / \psi$ is regular at $z= \pm \infty$. When (4.14) is satisfied, the r.h.s. of (4.13) is analytic everywhere in the whole complex plane and thus must be a constant by the Liouville theorem. Therefore the Bethe ansatz equation (4.14) is not only necessary but also sufficient condition for the r.h.s. of (4.13) to be independent of $z$.

To get the corresponding eigenvalue $E$, we consider the leading order expansion of $\psi(z)$

$$
\psi(z)=z^{M}-z^{M-1} \sum_{i=1}^{M} \alpha_{i}+\cdots
$$

It is easy to show that $\mathcal{Q}_{ \pm, 0} \psi(z)$ have the expansions,

$$
\begin{aligned}
\mathcal{Q}_{+} \psi & =-z^{M} \frac{(\sqrt{r})^{r}}{(\sqrt{s})^{s}}\left[\prod_{j=1}^{r}\left(q_{2}+1-\frac{(j-1) r+1}{r^{2}}\right)\right] \sum_{i=1}^{M} \alpha_{i}+\cdots, \\
\mathcal{Q}_{-} \psi & =z^{M-1} \frac{(\sqrt{s})^{s}}{(\sqrt{r})^{r}} \prod_{j=1}^{s}\left(2 l-q_{2}-\frac{(j-1) s+1}{s^{2}}\right)+\cdots, \\
\mathcal{Q}_{0} \psi & =z^{M}\left(l-q_{2}\right)+\cdots .
\end{aligned}
$$

Substituting these expressions into the Hamiltonian equation (4.5) and equating the $z^{M}$ terms, we arrive at

$$
E=w_{11}\left(s\left(2 l-q_{2}\right)-\frac{1}{s}\right)^{2}+w_{22}\left(r q_{2}-\frac{1}{r}\right)^{2}
$$




$$
\begin{aligned}
& +2 w_{12}\left(s\left(2 l-q_{2}\right)-\frac{1}{s}\right)\left(r q_{2}-\frac{1}{r}\right) \\
& +w_{1}\left(s\left(2 l-q_{2}\right)-\frac{1}{s}\right)+w_{2}\left(r q_{2}-\frac{1}{r}\right) \\
& -g\left[\prod_{j=1}^{r} r\left(q_{2}+1-\frac{(j-1) r+1}{r^{2}}\right)\right] \sum_{i=1}^{M} \alpha_{i},
\end{aligned}
$$

where $\left\{\alpha_{i}\right\}$ satisfy the Bethe ansatz equations (4.14). This gives the eigenvalue of the 2-mode boson Hamiltonian (1.1) with the corresponding eigenfunction $\psi(z)$ (4.8).

\section{Explicit examples corresponding to BECs}

We will now work out in complete detail the Bethe ansatz equations and energy eigenvalues of the Hamiltonian (1.1) for the special cases of $s, r \leq 2$ and $r=s=3$. These models arise in the description of Josephson tunneling effects and atom-molecule conversion processes in the context of BECs.

A. $s=1, r=1$

The Hamiltonian is

$$
H=\sum_{i}^{2} w_{i} N_{i}+\sum_{i, j}^{2} w_{i j} N_{i} N_{j}+g\left(a_{1}^{\dagger} a_{2}+a_{1} a_{2}^{\dagger}\right) .
$$

This is the so-called two coupled BEC model and has been solved in [27] via a different method, i.e. the ABA method. From the general results in the preceding section, in this case, we have $q_{1}=q_{2}=1$, which means that $2 l-q_{1}-q_{2}=2(l-1)=0,1, \cdots$. That is $l-1=0, \frac{1}{2}, 1, \cdots$. The differential operator representation of the Hamiltonian (5.1) is

$$
H=P_{2}(z) \frac{d^{2}}{d z^{2}}+P_{1}(z) \frac{d}{d z}+P_{0}(z)
$$

where

$$
\begin{aligned}
& P_{2}(z)=A_{11} z^{2} \\
& P_{1}(z)=-g z^{2}+B_{11} z+g \\
& P_{0}(z)=2(l-1) g z+D_{11}
\end{aligned}
$$

with

$$
\begin{aligned}
& A_{11}=w_{11}+w_{22}-2 w_{12} \neq 0 \\
& B_{11}=w_{1}-w_{2}+w_{11}+(5-4 l) w_{22}+(4 l-6) w_{12} \\
& D_{11}=2(l-1) w_{2}+4(l-1)^{2} w_{22} .
\end{aligned}
$$


The Bethe ansatz equations are given by

$$
\sum_{i \neq p}^{2(l-1)} \frac{2}{\alpha_{i}-\alpha_{p}}=\frac{g+B_{11} \alpha_{p}-g \alpha_{p}^{2}}{A_{11} \alpha_{p}^{2}}, \quad p=1,2, \cdots, 2(l-1)
$$

and the energy eigenvalues are

$$
E=4 w_{11}(l-1)^{2}+2 w_{1}(l-1)-g \sum_{i=1}^{2(l-1)} \alpha_{i}
$$

B. $s=2, r=1$

The Hamiltonian is

$$
H=\sum_{i}^{2} w_{i} N_{i}+\sum_{i, j}^{2} w_{i j} N_{i} N_{j}+g\left(a_{1}^{\dagger 2} a_{2}+a_{1}^{2} a_{2}^{\dagger}\right) .
$$

This is the homo-atomic-molecular BEC model and has been solved by the ABA method [27]. Specializing the general results in the preceding section to this case, we have $q_{1}=\frac{1}{4}$ or $\frac{3}{4}$, and $q_{2}=1$. The differential operator representation of the Hamiltonian (5.7) is thus

$$
H=P_{2}(z) \frac{d^{2}}{d z^{2}}+P_{1}(z) \frac{d}{d z}+P_{0}(z)
$$

where

$$
\begin{aligned}
& P_{2}(z)=A_{21} z^{2}+4 g z \\
& P_{1}(z)=-g z^{2}+B_{21} z+8 g q_{1} \\
& P_{0}(z)=g\left(2 l-q_{1}-1\right) z+D_{21}
\end{aligned}
$$

with

$$
\begin{aligned}
A_{21}= & 4 w_{11}+w_{22}-4 w_{12} \\
B_{21}= & 2 w_{1}-w_{2}+2 w_{11}\left(1+4 q_{1}\right)+w_{22}\left(3+2 q_{1}-4 l\right) \\
& +w_{12}\left(-7-8 q_{1}+8 l\right) \\
D_{21}= & 2 w_{1}\left(q_{1}-\frac{1}{4}\right)+w_{2}\left(2 l-q_{1}-1\right) \\
& +4 w_{11}\left(q_{1}-\frac{1}{4}\right)^{2}+w_{22}\left(2 l-1-q_{1}\right)^{2} \\
& +4 w_{12}\left(q_{1}-\frac{1}{4}\right)\left(2 l-1-q_{1}\right) .
\end{aligned}
$$

The Bethe ansatz equations are

$$
\sum_{i \neq p}^{2 l-1-q_{1}} \frac{2}{\alpha_{i}-\alpha_{p}}=\frac{8 g q_{1}+B_{21} \alpha_{p}-g \alpha_{p}^{2}}{\alpha_{p}\left(A_{21} \alpha_{p}+4 g\right)}, \quad p=1,2, \cdots, 2 l-1-q_{1}
$$


and the energy eigenvalues are given by

$$
E=2 w_{1}\left(2 l-\frac{5}{4}\right)+4 w_{11}\left(2 l-\frac{5}{4}\right)^{2}-g \sum_{i=1}^{2 l-1-q_{1}} \alpha_{i}
$$

C. $s=2, r=2$

The Hamiltonian is

$$
H=\sum_{i}^{2} w_{i} N_{i}+\sum_{i, j}^{2} w_{i j} N_{i} N_{j}+g\left(a_{1}^{\dagger 2} a_{2}^{2}+a_{1}^{2} a_{2}^{\dagger 2}\right) .
$$

This gives another model of the atom-molecule BECs. To our knowledge, this model has not been exactly solved previously. Applying the general results in the preceding section, we have in this case $q_{1}=\frac{1}{4}, \frac{3}{4}$ and $q_{2}=\frac{1}{4}, \frac{3}{4}$. The differential operator representation of the Hamiltonian (5.13) is

$$
H=P_{2}(z) \frac{d^{2}}{d z^{2}}+P_{1}(z) \frac{d}{d z}+P_{0}(z)
$$

where

$$
\begin{aligned}
& P_{2}(z)=4 g z^{3}+4 A_{22} z^{2}+4 g z, \\
& P_{1}(z)=B_{22} z^{2}+D_{22} z+8 g q_{1}, \\
& P_{0}(z)=F_{22} z+G_{22},
\end{aligned}
$$

with

$$
\begin{aligned}
A_{22}= & w_{11}+w_{22}-2 w_{12} \\
B_{22}= & 8 g\left(1+q_{1}-2 l\right) \\
D_{22}= & 2 w_{1}-2 w_{2}+2 w_{11}\left(1+4 q_{1}\right)+2 w_{22}\left(3-8 l+4 q_{1}\right) \\
& +8 w_{12}\left(-1-2 q_{1}+2 l\right) \\
F_{22}= & 4 g\left(2 l-q_{1}-\frac{1}{4}\right)\left(2 l-q_{1}-\frac{3}{4}\right) \\
G_{22}= & 2 w_{1}\left(q_{1}-\frac{1}{4}\right)+2 w_{2}\left(2 l-q_{1}-\frac{1}{4}\right)+4 w_{11}\left(q_{1}-\frac{1}{4}\right)^{2} \\
& +8 w_{12}\left(q_{1}-\frac{1}{4}\right)\left(2 l-q_{1}-\frac{1}{4}\right)+4 w_{22}\left(2 l-q_{1}-\frac{1}{4}\right)^{2} .
\end{aligned}
$$

Note that $\left(2 l-q_{1}-q_{2}\right)\left(2 l-q_{1}+q_{2}-1\right) \equiv\left(2 l-q_{1}-1 / 4\right)\left(2 l-q_{1}-3 / 4\right)$ for $q_{2}=1 / 4,3 / 4$. The Bethe ansatz equations read

$$
\sum_{i \neq p}^{2 l-q_{1}-q_{2}} \frac{2}{\alpha_{i}-\alpha_{p}}=\frac{8 g q_{1}+D_{22} \alpha_{p}-B_{22} \alpha_{p}^{2}}{4 \alpha_{p}\left(g \alpha_{p}^{2}+A_{22} \alpha_{p}+g\right)}, \quad p=1,2, \cdots, 2 l-q_{1}-q_{2}
$$


and the energy eigenvalues are

$$
\begin{aligned}
E= & 4 w_{11}\left(2 l-q_{2}-\frac{1}{4}\right)^{2}+4 w_{22}\left(q_{2}-\frac{1}{4}\right)^{2} \\
& +8 w_{12}\left(2 l-q_{2}-\frac{1}{4}\right)\left(q_{2}-\frac{1}{4}\right)+2 w_{1}\left(2 l-q_{2}-\frac{1}{4}\right) \\
& +2 w_{2}\left(q_{2}-\frac{1}{4}\right)-4 g\left(q_{2}+\frac{1}{4}\right)\left(q_{2}+\frac{3}{4}\right) \sum_{i=1}^{2 l-q_{1}-q_{2}} \alpha_{i} .
\end{aligned}
$$

D. $s=3, r=3$

The considered examples with $s, r \leq 2$ may in principle be treated using the ABA method based on Lie algebra $\mathrm{su}(2)$ (without any polynomial deformations). We now present an explicit example for which the ABA method is not applicable. The Hamiltonian is

$$
H=\sum_{i}^{2} w_{i} N_{i}+\sum_{i, j}^{2} w_{i j} N_{i} N_{j}+g\left(a_{1}^{\dagger 3} a_{2}^{3}+a_{1}^{3} a_{2}^{\dagger 3} .\right) .
$$

This is a non-linear optical model with third-order harmonic generation. Specializing the general results in the preceding section to this case, we have $q_{1}, q_{2}=\frac{1}{9}, \frac{4}{9}$, or $\frac{7}{9}$. The differential operator representation of the Hamiltonian (5.19) is

$$
H=P_{3}(z) \frac{d^{3}}{d z^{3}}+P_{2}(z) \frac{d^{2}}{d z^{2}}+P_{1}(z) \frac{d}{d z}+P_{0}(z),
$$

where

$$
\begin{aligned}
& P_{3}(z)=27 g\left(-z^{4}+z^{2}\right) \\
& P_{2}(z)=A_{33} z^{3}+B_{33} z^{2}+D_{33} z \\
& P_{1}(z)=F_{33} z^{2}+G_{33} z+K_{33} \\
& P_{0}(z)=R_{33} z+S_{33}
\end{aligned}
$$

with

$$
\begin{aligned}
A_{33}= & 9 g\left(18 l-9 q_{1}-13\right), \\
B_{33}= & 9\left(w_{11}+w_{22}-2 w_{12}\right), \\
D_{33}= & 9 g\left(9 q_{1}+5\right) \\
F_{33}= & 9 g\left(-36 l^{2}-\frac{76}{9}+34 l+36 l q_{1}-9 q_{1}^{2}-17 q_{1}\right), \\
G_{33}= & 3 w_{1}-3 w_{2}+w_{11}\left(7+18 q_{1}\right)+2 w_{12}\left(-9-18 q_{1}+18 l\right)+w_{22}\left(7+18 q_{1}\right), \\
K_{33}= & 9 g\left(q_{1}+9 q_{1}^{2}+\frac{4}{9}\right), \\
R_{33}= & 27 g\left(2 l-q_{1}-\frac{1}{9}\right)\left(2 l-q_{1}-\frac{4}{9}\right)\left(2 l-q_{1}-\frac{7}{9}\right), \\
S_{33}= & 9 w_{11}\left(q_{1}-\frac{1}{9}\right)^{2}+9 w_{22}\left(2 l-q_{1}-\frac{1}{9}\right)^{2}+18 w_{12}\left(q_{1}-\frac{1}{9}\right)\left(2 l-q_{1}-\frac{1}{9}\right) \\
& +3 w_{1}\left(q_{1}-\frac{1}{9}\right)+3 w_{2}\left(2 l-q_{1}-\frac{1}{9}\right) .
\end{aligned}
$$


The Bethe ansatz equations read

$$
\begin{gathered}
\sum_{i<j \neq p}^{2 l-q_{1}-q_{2}} \frac{162 g\left(\alpha_{p}^{4}-\alpha_{p}^{2}\right)}{\left(\alpha_{i}-\alpha_{p}\right)\left(\alpha_{j}-\alpha_{p}\right)}+\sum_{i \neq p}^{2 l-q_{1}-q_{2}} \frac{2\left(A_{33} \alpha_{p}^{3}+B_{33} \alpha_{p}^{2}+D_{33} \alpha_{p}\right)}{\alpha_{i}-\alpha_{p}} \\
=F_{33} \alpha_{p}^{2}+G_{33} \alpha_{p}+K_{33}, \quad p=1,2, \cdots, 2 l-q_{1}-q_{2}
\end{gathered}
$$

and the energy eigenvalues are

$$
\begin{aligned}
E= & 9 w_{11}\left(2 l-q_{2}-\frac{1}{9}\right)^{2}+9 w_{22}\left(q_{2}-\frac{1}{9}\right)^{2} \\
& +18 w_{12}\left(2 l-q_{2}-\frac{1}{9}\right)\left(q_{2}-\frac{1}{9}\right)+3 w_{1}\left(2 l-q_{2}-\frac{1}{9}\right) \\
& +3 w_{2}\left(q_{2}-\frac{1}{9}\right)-27 g\left(q_{2}+\frac{2}{9}\right)\left(q_{2}+\frac{5}{9}\right)\left(q_{2}+\frac{8}{9}\right) \sum_{i=1}^{2 l-q_{1}-q_{2}} \alpha_{i} .
\end{aligned}
$$

\section{Spectral Equivalence with QES Schrödinger Poten- tials}

The Hamiltonians in section 5 correspond to second order differential operators and can be mapped to Schrödinger equations with QES potentials via a suitable similarity transformation and change of variables [28].

Explicitly, if $H$ is written in the following form

$$
H=P(z) \frac{d^{2}}{d z^{2}}+\left(Q(z)+\frac{1}{2} P^{\prime}(z)\right) \frac{d}{d z}+R(z)
$$

then it can be mapped to a Schrödinger operator,

$$
\tilde{H}=-e^{W(x)} H e^{-W(x)}=-\frac{d^{2}}{d x^{2}}+V(x),
$$

where the variable $x$ and $z$ are related by (we assume $z=z(x)$ is invertible on a certain interval to give $x=x(z)$ ) [29],

$$
x=x(z)= \pm \int^{z} \frac{d y}{\sqrt{P(y)}}
$$

and $W(x)$ is given as

$$
W(x)=\int^{z(x)} \frac{Q(y)}{2 P(y)} d y .
$$

The potential function is given by

$$
V(x)=\left.\left\{-R(z)+\frac{1}{2} Q^{\prime}(z)-\frac{Q(z)\left(P^{\prime}(z)-Q(z)\right)}{4 P(z)}\right\}\right|_{z=z(x)}
$$


Then the solutions of the 2nd order ODE $H \psi(z)=E \psi(z)$ with eigenvalue $E$ is mapped to solutions of the Schrödinger equation

$$
\tilde{H} \tilde{\psi}(x)=\tilde{E} \tilde{\psi}(x)
$$

with eigenvalue $\tilde{E}=-E$ and corresponding Schrödinger wavefunction

$$
\tilde{\psi}(x)=e^{-W(x)} \psi(z(x)) .
$$

We will not discuss the square integrability of the Schrödinger wavefunction $\tilde{\psi}(x)$, but derive the explicit Schrödinger potentials corresponding to the special models in the preceding section.

I. $s=1, r=1$ :

For this case we have

$$
\begin{aligned}
& P(z)=A_{11} z^{2} \\
& Q(z)=-g z^{2}+\left(B_{11}-A_{11}\right) z+g \\
& R(z)=2(l-1) g z+D_{11} .
\end{aligned}
$$

From (6.3), we obtain

$$
z(x)=e^{\sqrt{A_{11}} x}
$$

The potential is

$$
\begin{aligned}
V(x)= & \frac{\left(g z^{2}+\left(A_{11}-B_{11}\right) z-g\right)\left(g z^{2}+\left(3 A_{11}-B_{11}\right) z-g\right)}{4 A_{11} z^{2}} \\
& -g(2 l-1) z+\frac{B_{11}-A_{11}}{2}-D_{11} \\
= & \frac{g^{2}}{2 A_{11}} \cosh \left(2 \sqrt{A_{11}} x\right)+g\left(2-\frac{B_{11}}{A_{11}}\right) \sinh \left(\sqrt{A_{11}} x\right) \\
& -(2 l-1) g e^{\sqrt{A_{11}} x}-D_{11}+\frac{\left(A_{11}-B_{11}\right)^{2}-2 g^{2}}{4 A_{11}} .
\end{aligned}
$$

II. $s=2, r=1$ :

In this case we have,

$$
\begin{aligned}
& P(z)=A_{21} z^{2}+4 g z \\
& Q(z)=-g z^{2}+\left(B_{21}-A_{21}\right) z+2 g\left(4 q_{1}-1\right) \\
& R(z)=g\left(2 l-q_{1}-1\right) z+D_{21}
\end{aligned}
$$

and $q_{1}=\frac{1}{4}$ or $\frac{3}{4}$. From (6.3), we derive

$$
z(x)=\frac{2 g}{A_{21}}\left(\cosh \left(\sqrt{A_{21}} x\right)-1\right) .
$$


The potential is

$$
\begin{aligned}
V(x)= & \frac{\left(g z^{2}+\left(A_{21}-B_{21}\right) z+2 g-8 g q_{1}\right)\left(g z^{2}+\left(3 A_{21}-B_{21}\right) z+6 g-8 g q_{1}\right)}{4\left(A_{21} z^{2}+4 g z\right)} \\
& -g\left(2 l-q_{1}\right) z+\frac{B_{21}-A_{21}}{2}-D_{21} \\
= & \frac{g^{2}}{A_{21}^{2}} \tanh ^{2}\left(\frac{\sqrt{A_{21}}}{2} x\right) \sinh ^{2}\left(\frac{\sqrt{A_{21}}}{2} x\right)\left[\frac{4 g^{2}}{A_{21}} \sinh ^{2}\left(\frac{\sqrt{A_{21}}}{2} x\right)+2 A_{21}-B_{21}\right] \\
& +\frac{1}{4 A_{21}}\left[\left(A_{21}-B_{21}\right)\left(3 A_{21}-B_{21}\right)+8 g^{2}\left(1-2 q_{1}\right)\right] \tanh ^{2}\left(\frac{\sqrt{A_{21}}}{2} x\right) \\
& -\frac{4\left(2 l-q_{1}\right) g^{2}}{A_{21}} \sinh ^{2}\left(\frac{\sqrt{A_{21}}}{2} x\right) \\
& +\frac{\left(3-8 q_{1}\right) A_{21}+\left(4 q_{1}-2\right) B_{21}}{4 \cosh ^{2}\left(\frac{\sqrt{A_{21}}}{2} x\right)}+\frac{B_{21}-A_{21}}{2}-D_{21} .
\end{aligned}
$$

Here we have used $\left(1-4 q_{1}\right)\left(3-4 q_{1}\right)=0$ for the two allowed $q_{1}$ values $q_{1}=\frac{1}{4}$ or $\frac{3}{4}$.

Let us consider the special case of $A_{21}=0$. In this case,

$$
z(x)=g x^{2}
$$

as can be seen from the $A_{21} \rightarrow 0$ limit of (6.12). The Schrödinger potential (6.13) then becomes

$$
V(x)=\frac{g^{4}}{16} x^{6}-\frac{g^{2}}{8} B_{21} x^{4}+\frac{B_{21}^{2}+8 g^{2}(1-4 l)}{16} x^{2}+q_{1} B_{21}-D_{21} .
$$

This is a non-singular sextic potential.

III. $s=2, r=2$ :

For this case we have

$$
\begin{aligned}
& P(z)=4 g z^{3}+4 A_{22} z^{2}+4 g z \\
& Q(z)=B_{22} z^{2}+\left(D_{22}-4 A_{22}\right) z+8 g q_{1}-2 g \\
& R(z)=F_{22} z+G_{22}
\end{aligned}
$$

and $q_{1}=\frac{1}{4}$ or $\frac{3}{4}$. From (6.3), we obtain

$$
z(x)=g^{-\frac{1}{3}} \wp\left(g^{\frac{1}{3}} x ; g_{2}, g_{3}\right)-\frac{A_{22}}{3 g},
$$

where $\wp\left(x ; g_{2}, g_{3}\right)$ is Weierstrass's elliptic function with invariants $g_{2}$ and $g_{3}$ given by

$$
g_{2}=\frac{4}{3} g^{\frac{2}{3}}\left(\frac{A_{22}^{2}}{g^{2}}-3\right), \quad g_{3}=\frac{4}{27} A_{22}\left(9-\frac{2 A_{22}^{2}}{g^{2}}\right) .
$$


Hereafter we will denote $\wp\left(x ; g_{2}, g_{3}\right)$ simply as $\wp(x)$. The potential is computed as follows

$$
\begin{aligned}
V(x)= & \left(B_{22} z^{2}+\left(D_{22}-4 A_{22}\right) z+8 g q_{1}-2 g\right) \\
& \times \frac{\left(B_{22}-12 g\right) z^{2}+\left(D_{22}-12 A_{22}\right) z+8 g q_{1}-6 g}{16\left(g z^{3}+A_{22} z^{2}+g z\right)} \\
& +\left(B_{22}-F_{22}\right) z+\frac{D_{22}-4 A_{22}}{2}-G_{22} \\
= & \sum_{i=1}^{4} c_{i} \frac{\left(g^{-\frac{1}{3}} \wp\left(g^{\frac{1}{3}} x\right)-\frac{A_{22}}{3 g}\right)^{i}}{4 \wp^{\prime}\left(g^{\frac{1}{3}} x\right)^{2}}+\left(B_{22}-F_{22}\right) g^{-\frac{1}{3}} \wp\left(g^{\frac{1}{3}} x\right) \\
& +\frac{A_{22}\left(B_{22}-F_{22}\right)}{3 g}+\frac{D_{22}-4 A_{22}}{2}-G_{22},
\end{aligned}
$$

where

$$
\begin{aligned}
& c_{1}=2 g\left(4 q_{1}-3\right)\left(D_{22}-4 A_{22}\right)+2 g\left(4 q_{1}-1\right)\left(D_{22}-12 A_{22}\right), \\
& c_{2}=2 g\left(4 q_{1}-3\right) B_{22}+2 g\left(4 q_{1}-1\right)\left(B_{22}-12 g\right)+\left(D_{22}-4 A_{22}\right)\left(D_{22}-12 A_{22}\right), \\
& c_{3}=B_{22}\left(D_{22}-12 A_{22}\right)+\left(B_{22}-12 g\right)\left(D_{22}-4 A_{22}\right), \\
& c_{4}=B_{22}\left(B_{22}-12 g\right) .
\end{aligned}
$$

Here we have used $4\left(g z^{3}+A_{22} z^{2}+g z\right)=4 \wp\left(g^{\frac{1}{3}} x\right)^{3}-g_{2} \wp\left(g^{\frac{1}{3}} x\right)-g_{3}=\wp^{\prime}\left(g^{\frac{1}{3}} x\right)^{2}$ and $\left(1-4 q_{1}\right)\left(3-4 q_{1}\right)=0$ for the two allowed $q_{1}$ values $q_{1}=\frac{1}{4}$ or $\frac{3}{4}$.

\section{Discussion}

Let us now quickly summarize the work. We began by constructing the boson representation of a class of $s u(1,1)$ polynomially deformed algebras $(2.1)$, deriving their infinite dimensional Fock space realization and lowest weight state parametrization. We then used the Jordan-Schwinger like construction to get the polynomial algebra (3.2) which possesses finite dimensional irreducible representations. We used the differential realization of (3.2) to rewrite the Hamiltonian (1.1) as QES differential operators acting on the finite dimensional monomial space. The exact eigenfunctions and eigenvalues of the Hamiltonian were then found by employing the Functional Bethe Ansatz technique. As examples, we provided some explicit expressions for the BEC models which correspond to the $r, s \leq 2$ cases of (1.1) and established the spectral correspondence of these specific models with QES Schrödinger potentials.

In deriving our results, we showed that in general the Hamiltonians defined in (1.1) are QES differential operator of order 3 or higher. This paper provides an algebraization of such higher order QES differential operators and unravels the dynamical polynomial algebra symmetry of (1.1). It also shows that the Functional Bethe Ansatz method provides a simple way to find exact eigenvalues and eigenfunctions of such higher order differential operators. 
There are a number of extensions that we plan to pursue in this line of investigation. Firstly, we note that the Jordan-Schwinger like construction can be extended straightforwardly to study other non-linear quantum optical models such as the general multi-mode boson Hamiltonians of the form

$$
\begin{aligned}
H= & \sum_{i}^{k+k^{\prime}} w_{i} N_{i}+\sum_{i, j}^{k+k^{\prime}} w_{i j} N_{i} N_{j} \\
& +g\left(a_{1}^{\dagger m_{1}} \cdots a_{k}^{\dagger m_{k}} a_{k+1}^{m_{k+1}} \cdots a_{k+k^{\prime}}^{m_{k+k^{\prime}}}+a_{1}^{m_{1}} \cdots a_{k}^{m_{k}} a_{k+1}^{\dagger m_{k+1}} \cdots a_{k+k^{\prime}}^{\dagger m_{k+k^{\prime}}}\right) .
\end{aligned}
$$

Results on on this and other models of physical interest will be presented elsewhere.

Acknowledgments: This work was supported by the Australian Research Council. The authors would like to thank Ryu Sasaki for very valuable comments and suggestions which lead to significant improvement of the presentation of the paper.

\section{Appendix}

In this appendix, we work out the expansion coefficients in front of $\frac{d^{i}}{d z^{i}}$ in the xpansion of $\prod_{i=1}^{m}\left(z \frac{d}{d z}+A_{i}\right)$.

First, we see

$$
\begin{aligned}
\prod_{i=1}^{m}\left(z \frac{d}{d z}+A_{i}\right)= & \prod_{i=1}^{m} A_{i}+\left(\sum_{j_{1}=1}^{m} \prod_{i \neq j_{1}}^{m} A_{i}\right) z \frac{d}{d z}+\left(\sum_{j_{1}<j_{2}}^{m} \prod_{i \neq j_{1} \neq j_{2}}^{m} A_{i}\right)\left(z \frac{d}{d z}\right)^{2} \\
& +\cdots+\left(\sum_{j_{1}<j_{2}<. .<j_{m}}^{m} \prod_{i \neq j_{1} \ldots \neq j_{m}}^{m} A_{i}\right)\left(z \frac{d}{d z}\right)^{m}
\end{aligned}
$$

and since

$$
\begin{aligned}
\left(z \frac{d}{d z}\right)^{k} & =z^{k} \frac{d^{k}}{d z^{k}}+\left(\sum_{n=1}^{k-1} n\right) z^{k-1} \frac{d^{k-1}}{d z^{k-1}}+\left(\sum_{n_{1}<n_{2}}^{k-1} n_{1}\left(n_{2}-1\right)\right) z^{k-2} \frac{d^{k-2}}{d z^{k-2}}+\cdots \\
& =\sum_{i=1}^{k} L_{k, i} z^{i} \frac{d^{i}}{d z^{i}}
\end{aligned}
$$

where

$$
\begin{aligned}
L_{k, k} & =1 \\
L_{k, i} & =\sum_{n_{1}<\ldots<n_{k-i}}^{k-1} n_{1}\left(n_{2}-1\right) \ldots\left(n_{k-i}-(k-i)+1\right), \quad i<k
\end{aligned}
$$

We can regroup the equation (8.1) as

$$
\prod_{i=1}^{m}\left(z \frac{d}{d z}+A_{i}\right)
$$




$$
\begin{aligned}
= & \prod_{i=1}^{m} A_{i}+\left(L_{1,1}\left(\sum_{j_{1}=1}^{m} \prod_{i \neq j_{1}}^{m} A_{i}\right)+\cdots+L_{m, 1}\left(\sum_{j_{1}<j_{2}<\cdots<j_{m}}^{m} \prod_{i \neq j_{1} \cdots \neq j_{m}}^{m} A_{i}\right)\right) z \frac{d}{d z} \\
& +\left(L_{2,2}\left(\sum_{j_{1}<j_{2}}^{m} \prod_{i \neq j_{1} \neq j_{2}}^{m} A_{i}\right)+\cdots+L_{m, 2}\left(\sum_{j_{1}<j_{2}<\cdots<j_{m}}^{m} \prod_{i \neq j_{1} \cdots \neq j_{m}}^{m} A_{i}\right)\right) z^{2} \frac{d^{2}}{d z^{2}} \\
& + \text { higher order terms } \\
=\prod_{i=1}^{m} A_{i} & +\sum_{i=1}^{m} \sum_{k=i}^{m}\left(\sum_{l_{1}<\ldots<l_{k}}^{m} \prod_{j \neq l_{1} \neq \cdots \neq l_{k}}^{m} A_{j}\right) L_{k, i} z^{i} \frac{d^{i}}{d z^{i}}
\end{aligned}
$$

\section{References}

[1] P.W. Higgs, J. Phys. A: Math. Gen. 12, 309 (1979).

[2] M. Rocek, Phys. Lett. B 255, 554 (1991).

[3] K Schoutens, A. Sevrin and P. Van Nieuwenhuizen, Comm. Math. Phys. 124, 87 (1991); Phys. Lett. B bf 255, 549 (1991).

[4] Ya.I. Granovsky, A.S. Zhedanov and I.M. Lutzenko, Ann. Phys. (NY) 217, 1 (1992).

[5] P. Letourneau and L. Vinet, Ann. Phys. (NY) 243, 144 (1995).

[6] C. Quesne, Phys. Lett. A 193, 249 (1994); SIGMA 3, Paper 067 (2007).

[7] D. Bonatsos, C. Daskaloyannis and K. Kokkotas, Phys. Rev. A 50, 3700 (1994).

[8] V.P. Karassiov and A. Klimov, Phys. Lett. A 191, 117 (1994).

[9] V.P. Karassiov, A.A. Gusev and S.I. Vinitsky, Phys. Lett. A 295, 247 (2002).

[10] S.M. Klishevich and M.S. Plyushchay, Nucl. Phys. B 606, 583 (2001); ibid 616, 403 (2001).

[11] S.P. Smith, Trans. Amer. Math. Soc. 322, 285 (1990).

[12] A.S. Zhedanov, Mod. Phys. Lett. A 7, 507 (1992).

[13] V.S. Kumar, B.A. Bambah, and R. Jagannathan, J. Phys. A: Math. Gen. 38, 34 (2001); Mod. Phys. Lett. A 17, 1559 (2002).

[14] J. Beckers, Y. Brihaye, and N. Debergh, J. Phys. A: Math. Gen. 32, 2791 (1999).

[15] N. Debergh, J. Phys. A: Math. Gen. 33, 7109 (2000).

[16] A. Turbiner, Comm. Math. Phys. 118, 467 (1988); Quasi-exactly-solvable differential equations, 1994, hep-th/9409068. 
[17] A.G. Ushveridze, Quasi-exactly solvable models in quantum mechanics, Institute of Physics Publishing, Bristol, 1994.

[18] A. Gonzárez-López, N. Kamran and P. Olver, Comm. Math. Phys. 153, 117 (1993).

[19] P.B. Wiegmann and A.V. Zabrodin, Phys. Rev. Lett. 72, 1890 (1994); Nucl. Phys. B 451, 699 (1995).

[20] R. Sasaki, W.-L. Yang, and Y.-Z. Zhang, SIGMA 5, Paper 104 (2009).

[21] M.H. Anderson, J.R. Ensher, M.R. Matthews, C.E. Wieman and E.A. Cornell, Science 269, 198 (1995).

[22] J.R. Anglin and W. Ketterle, Nature 416, 211 (2002).

[23] P. Zoller, Nature 417, 493 (2002).

[24] E.A. Donley, N.R. Claussen, S.T. Thompson and C.E. Wieman, Nature 417, 529 (2002).

[25] G. Álvarez, F. Finkel, A. González-López and M.A. Rodríguez, J. Phys. A: Math. Gen. 35, 8705 (2002).

[26] G. Álvarez and R.F. Álvarez-Estrada, J. Phys. A: Math. Gen. 28, 5767 (1995); ibid 34, 10045 (2001).

[27] J. Links, H.-Q. Zhou, R.H. McKenzie, and M.D. Gould, J. Phys. A: Math. Gen. 36, R63 (2003).

[28] O.B. Zaslavskii, Phys. Lett. A 149, 365 (1990).

[29] D. Gomez-Ullate, N. Kamran, and R. Milson, J. Phys. A: Math. Gen. 38, 2005 (2005). 